\title{
Revisión sistemática de nuevos anticoagulantes orales frente a warfarina en fibrilación auricular no valvular
}

Systematic review of new oral anticoagulants versus warfarin in non valvular atrial fibrillation

\author{
José Alejandro Mendoza (1), Federico Arturo Silva (1), Carlos Hernando Ortiz Castro (1), Lady Marcela Rangel (1)
}

\begin{abstract}
RESUMEN
INTRODUCCión: los nuevos anticoagulantes orales son el más reciente avance en anticoagulación oral. Existen suficientes estudios publicados, pero muy poca síntesis de la información.

Métodos: se realizó una búsqueda sistemática de los estudios en Medline, Cochrane y Embase con los términos predefinidos. Se aplicaron los criterios de inclusión y exclusión definidos, se definieron las variables utilizadas en este estudio según el beneficio (reducción de la mortalidad y reducción del ACV) y el riesgo en la anticoagulación (sangrado intracraneal, sangrado gastrointestinal, sangrado mayor y otros sangrados). Se seleccionaron los artículos y se extrajo la información en una hoja de cálculo para análisis.

REsultados: se incluyeron 24 meta-análisis y 62 subanálisis; 77 \% de los meta-análisis mostraron reducción de ACV, $65 \%$ mortalidad, $71 \%$ reportó disminución de hemorragia intracraneal y $45 \%$ de sangrado mayor, pero hubo aumento de sangrado gastrointestinal en el 5 \% de los estudios. En relación a los resultados de los subanálisis, 4 estudios mostraron algún dato estadísticamente significativo para apixabán, 5 para dabigatrán, 3 para edoxabán y 5 estudios para rivaroxabán. Existen diferencias en algunas variables como sexo, riesgo de $\mathrm{ACV}$, resultados a 30 días, función renal; diferentes dosis (dabigatrán y edoxabán), según la edad, síntomas gastrointestinales, relación con el INR; niveles séricos del medicamento, uso de amiodarona, uso previo de warfarina; enfermedad arterial periférica, transición a warfarina; factores de riesgo de HGI y sangrado mayor para cada anticoagulante en particular.
\end{abstract}

ConClusiones: los nuevos anticoagulantes orales muestran mejor perfil de seguridad y de eficacia frente a warfarina.

PALABRAS CLAVES: accidente cerebrovascular, fibrilación auricular (DeCS).

\section{Contribución de los AUTOREs}

José Alejandro Mendoza, Federico Arturo Silva, Carlos Hernando Ortiz Castro, Lady Marcela Rangel: análisis de resultados, elaboración de la estructura del artículo y discusión de resultados.

\section{SUMMARY}

INTRODUCTION: New oral anticoagulants are the latest advancement in oral anticoagulation. Several studies are published, but a few studies of synthesis information.

Methods: A systematic review of the studies in MEDLINE, COCHRANE and EMBASE was performed with the predefined terms. The defined inclusion and exclusion criteria were applied; variables used in this study were defined according to benefits (reduction of mortality and reduction of stroke) and anticoagulation risks (intracranial bleeding, gastrointestinal bleeding, major bleeding and other bleeding). Articles were selected and information was extracted into a spreadsheet for analysis.

REsult: Were included 24 meta-analyzes and 62 sub-analyzes; $77 \%$ of meta-analyzes showed stroke reduction, $65 \%$ in mortality, $71 \%$ reported decreased of intracranial hemorrhage and $45 \%$ in major bleeding, but there was an increase in gastrointestinal bleeding in 54\% of the studies. Regarding the results of the sub-analysis, 4 studies for apixaban, 5 for dabigatran, 3 for edoxaban and 5 studies for rivaroxaban showed some statistically

(1) Grupo de Ciencias Neurovasculares, Fundación Cardiovascular de Colombia, Hospital Internacional de Colombia, Piedecuesta, Santander.

Recibido 6/02/17. Aceptado: 18/07/17.

Correspondencia: José Alejandro Mendoza, jmendoza45@msn.com 
significant data. There are differences in some variables such as sex, stroke risk, 30-day results, renal function; Different doses (dabigatran and Edoxaban), according to age, gastrointestinal symptoms, relation with INR; Serum levels of the drug, use of amiodarone, prior use of warfarin; Peripheral arterial disease, transition to warfarin; Risk factors for gastrointestinal bleeding and major bleeding for each particular anticoagulant.

Conclusion: The new oral anticoagulants shows better safety and efficacy profile against Warfarin.

KEY WORDS: Stroke, atrial fibrillation, benefit risk assessment, systematic review.(MeSH).

\section{INTRODUCCIÓN}

La fibrilación auricular (FA) es la arritmia cardiaca más frecuente y es un factor de riesgo para ataque cerebrovascular (ACV) (1). La incidencia de ACV en pacientes con FA es de $6,2 \%$ anual en mujeres y $4,2 \%$ en hombres ( 2 ) y los factores de riesgo descritos para ACV en personas con FA son el ACV previo o isquemia cerebral transitoria (AIT), hipertensión arterial, diabetes, edad avanzada, falla cardiaca, sexo femenino, enfermedad vascular periférica y falla renal crónica (3). La terapia anticoagulante es recomendada para la prevención de ACV/tromboembolismo sistémico en FA. Se han descrito diferentes riesgos de la anticoagulación oral, como gastritis, que lleva a la interrupción del tratamiento hasta el sangrado intracraneal y es considerada el evento adverso de mayor impacto clínico después de la muerte por cualquier evento adverso (4).

La warfarina ha mostrado eficacia en la reducción el riesgo de ACV (5). Sin embargo, son conocidas las limitaciones de su uso como el estrecho rango terapéutico y la fluctuación del mismo, el riesgo de sangrado por sobreanticoagulación, las interacciones con medicamentos, alimentos y la necesidad de monitoreo regular del INR. Los nuevos anticoagulantes orales (NACO) apixabán, dabigatrán, rivaroxabán y edoxabán, son una alternativa a la warfarina. Diferentes meta-análisis y subanálisis de los ensayos clínicos pivotales han sido publicados, pero no existe una síntesis de toda esta información publicada (6).

A partir de los estudios publicados se realizó un análisis cualitativo tratando de sintetizar toda la información disponible sobre los nuevos anticoagulantes orales frente warfarina en fibrilación auricular no valvular. Así mismo se describirán los resultados de la terapia anticoagulante en subgrupos de pacientes no analizados ni reportados en los ensayos clínicos pivótales en los cuales existan datos estadísticamente significativos.

\section{MATERIALES Y MÉTODOS}

Se realizó una búsqueda sistemática de los meta-análisis, ensayos clínicos, revisiones sistemáticas, y subanálisis de ensayos clínicos, en los cuales se compararon los nuevos anticoagulantes con warfarina en FA no valvular publicados desde el año 2009 hasta octubre de 2016. La búsqueda se realizó en Medline, Cochrane, Embase, se realizó la búsqueda en octubre de 2016 utilizando los términos: "atrial fibrillation", "stroke", "warfarin", "apixabán", "rivaroxabán", "dabigatrán” y "edoxabán”, "sistematic review”, "meta-analysis" "clinical study", "clinical trial", se utilizaron los operadores de sintaxis AND, OR y NOT. Se realizó una búsqueda manual en "bola de nieve" utilizando las referencias bibliográficas de los artículos identificados. Se excluyeron términos como: "ablation surgery", "atrial ablation" o "heparin". Para realizar la búsqueda de los subestudios de los ensayos clínicos pivótales se revisaron uno por uno todas las referencias para cada anticoagulante filtrado por (clinical study y clinical trial). Los criterios de inclusión fueron: meta-análisis, subaanálisis, inclusión de warfarina como comparador, terapia anticoagulante en fibrilación auricular. Criterios de exclusión: ablación sinusal, válvula mecánica, trombosis venosa profunda, otras trombosis.

Se realizó una exploración previa de las guías publicadas en anticoagulación oral con el fin de definir el contexto de decisión que para este caso fue definido como las variables a ser evaluadas, se utilizaron las guías americana y europea para el manejo de pacientes con fibrilación auricular, según la evaluación de las guías los autores escogieron incluir los criterios para beneficios y riesgos de la anticoagulación oral (reducción de la muerte, disminución de ACV, hemorragia intracraneal, hemorragia gastrointestinal, sangrado mayor y otros) $(7,8)$.

Uno de los autores seleccionó los títulos y los resúmenes de los estudios identificados. Se excluyeron aquellos estudios que no se consideraron relevantes para esta revisión, finalmente se incluyeron los artículos después de leer los resúmenes, no se incluyeron los ensayos clínicos pivótales ya que los resultados fueron incluidos en todos los metaanálisis incluidos en la revisión. No se incluyeron aquellos subanálisis que no se consideraran pertinentes o que no reportaran alguna de las variables previamente definidas en la revisión. Se utilizó la metodología PRISMA en la cual se evaluaron: el título, resumen, objetivo; protocolo, búsqueda y evaluación de riesgo de sesgo. Se utilizó el software Review Manager versión 5.3. 


\section{RESULTADOS}

La búsqueda de los meta-análisis identificó 254 estudios de los cuales 76 se escogieron para evaluación, la búsqueda de los subestudios identificó: 114 estudios para apixabán, 187 para dabigatrán, 231 para rivaroxabán y 56 para edoxabán. Se incluyeron 24 meta-análisis, 22 subanálisis de apixabán, 16 de dabigatrán, 5 de edoxabán y 19 de rivaroxabán (figura 1), en total se incluyeron 86 artículos entre meta-análisis y subestudios. Los artículos finalmente seleccionados fueron ingresados en una hoja de datos diseñada con el objetivo de sintetizar los resultados de los estudios, así como organizar la información recolectada. Tres de los autores revisaron cada uno de los artículos con el fin de identificar y llenar en la hoja de cálculo los datos considerados estadísticamente significativos (intervalos de confianza que no cruzaran 1 , o $\mathrm{p}<0,05)$.

\section{Resultados de los meta-análisis}

Se incluyeron 24 artículos que analizaron alguno de los nuevos anticoagulantes orales frente a warfarina para la prevención de ACV/embolismo sistémico en paciente con FA, no todos los estudios analizaron o incluyeron la totalidad de las variables definidas en esta revisión. La media del número de pacientes incluidos fue de 56,118 pacientes, $96 \%$ de los meta-análisis analizó algún resultado para dabigatrán, 92\% rivaroxabán; 88 \% apixabán y $46 \%$ edoxabán (tabla 1). Se describieron los meta-análisis si reportaron los resultados por eficacia y riesgo: todos reportaron resultados de seguridad y el $71 \%$ (17/25) reportaron de eficacia (algunos solo analizaron el riesgo de sangrado); los resultados fueron divididos para cada variable incluida: $77 \%(13 / 18)$ de los meta-análisis reportó resultados en disminución del riesgo de ACV/embolismo sistémico, $65 \%$ (10/17) disminución del riesgo de muerte, $71 \%$ (15/21) para disminución de hemorragia intracraneal, para disminución de sangrado mayor $45 \%$ (9/20), para hemorragia gastrointestinal 13 meta-análisis reportaron algún resultado para esta variable, de los cuales 7 reportaron aumento de riesgo con NACO (54\%) y uno comparó apixabán versus dabigatrán (Baker y Col) (9), donde hubo reducción de riesgo de sangrado GI RR: 0,58 (IC 95 \% 0,41-0,82) con apixabán. Dos metaanálisis fueron realizados solo para comparar dabigatrán y warfarina (Romanelli y Col y Bloomy Col) $(10,11)$ y uno para rivaroxabán que fue el estudio de wasserlauf y Col (12), que reportó disminución en el riesgo de sangrado fatal con rivaroxabán RR: 0,48 (IC 95 \% 0,31-0,74). El meta-análisis de Gomez y Col (13), reportó resultados estadísticamente significativos de disminución del riesgo de muerte, ACV/ embolismo sistémico, HIC y sangrado mayor con apixabán, dabigatrán y rivaroxabán en tiempo en rango terapéutico de warfarina (TRT) $<65 \%$ de warfarina. Raccah y Col (14),

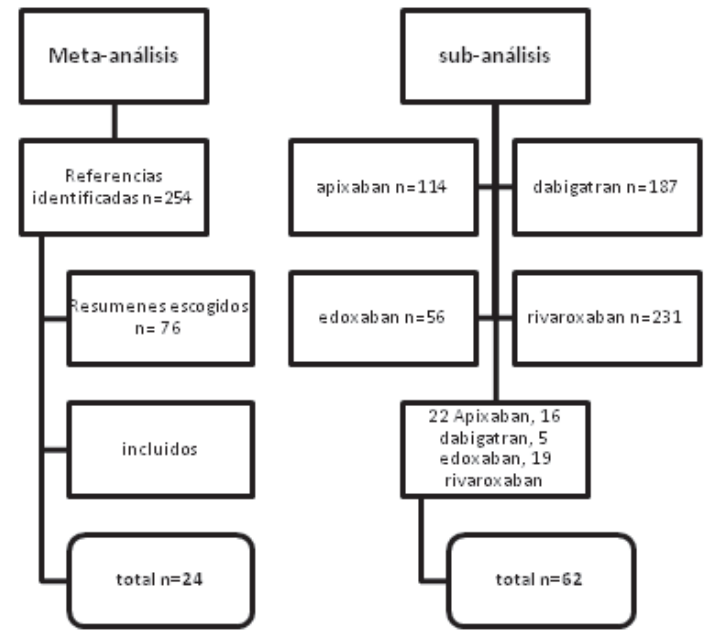

Figura 1. Artículos incluidos en la revisión

reportaron disminución del sangrado mayor con todos los $\mathrm{NACO}$ en pacientes con función renal moderada $(\mathrm{CrCl}$ 50-80) RR: 0,84 (IC 95 \% 0,78-0,91). Savarese y Col (15), reportaron aumento del riesgo de muerte y disminución de HIC en pacientes con falla cardiaca RR: 1,70 (IC 95\% 1,31-2,19 p<0,01) y RR: 0,74 (IC $95 \%$ 0,63-0,88).

\section{Resultados de los subanálisis}

Un total de 60 subanálisis de los ensayos clínicos pivótales fueron incluidos, 21 para apixabán, 15 dabigatrán, 5 edoxabán y 19 rivaroxabán (figura 1). Debido a la gran cantidad de artículos incluidos, solo se reportaron aquellos con resultados estadísticamente significativos (tabla 2), de los 21 subanálisis incluidos para apixabán solo 3 reportaron datos estadísticamente significativos; Vinereanu y Col (16) reportaron disminución de muerte por cualquier causa y disminución de muerte cardiovascular HR: 0,63 (IC 95 \% 0,55-0,73 P<0,0001) y HR: 0,62 (IC $95 \%$ 0,51-0,75 $\mathrm{P}<0,0001)$ respectivamente; Lopes y Col (17) reportaron disminución del riesgo de sangrado intracraneal, sangrado mayor y cualquier sangrado en relación con el CHADS2 Y CHAD2DS2VASC; HR: 0,42 (IC $95 \%$ 0,30-0,58 $\mathrm{P}<0,0001$ ), HR: 0,69; (IC $95 \%$ 0,60-0,80), HR: 0,71 (IC $95 \%$ 0,68-0,75 P<0,0001) respectivamente; Hylek y Col (18) reportaron disminución del sangrado mayor seguido de muerte a los 30 días y de sangrado intracraneal HR: 0,50 (0,33-0,74; IC 95 \%) P<0,001, 0,42 (0,30-0,58; IC 95 \%) $\mathrm{P}<0,001$.

Para dabigatrán 4 estudios mostraron alguna variable estadísticamente significativa; Brambatti y Col (19), reportaron disminución de riesgo de $\mathrm{ACV} /$ embolismo sistémico 
Tabla 1. Resumen de los meta-análisis incluidos en la revisión sistemática

Estudio

40777

Slot \& $\operatorname{col}(34)$

$\begin{array}{ll}\text { Assiri y col (35) } & 80906 \\ \text { Wasserlauf y col (36) } & 23063\end{array}$

Baker y col (37)

44733

Testa y col (38)

50578

Miller y col (39)

44563

Kwong y col (40)

61406

Dentali y col (41)

54875

Holster y col (42)

151578

Dogliotti y col (43)

51895

Romanelli y col (44)

348750

Gómez A y col (13) 50578

Loffredo y col (45)

71302

Raccah y col (46)

94897

Lin y col (47)

897948

Jía B y col (48)

72961

Bloom BJ y col (11) 20332

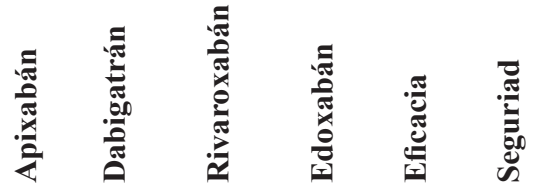

Mortalidad /ACV/embolismo /HIC/HGI/

Sangrado mayor
Mortalidad: OR 0,89 IC 0,82-0,97 ACV/

Embolismo: OR 0,81 IC 0,72-0,91 HIC: OR 0,56

IC 0,45-0,70 Sangrado mayor: OR 0,89 IC 0,810,98

NI

$\mathrm{x} \quad \mathrm{x} \quad \mathrm{NI}$

$\mathrm{x} \quad \mathrm{NI}$

$\mathrm{x} \quad \mathrm{x}$

ACV/embolismo: RR 0,67 IC 0,48-0,9 HGI: RR 0,58 IC 0,41-0,82 Sangrado mayor: RR 0,74 IC $0,60-0,91$

Mortalidad: OR 0,90 IC 0,84-0,96 ACV/ embolismo: OR 0,64 IC 0,44-0,94 HIC: OR 0,43 IC $0,34-0,55$

Mortalidad: RR 0,88 IC 0,82-0,95 ACV/ embolismo: RR 0,87 IC 0,67-0,92 HIC: RR 0,49 IC 0,36 - 0,66

ACV/embolismo: RR 0,71 IC 0,54-0.92 Sangrado mayor: RR 0,88 IC 0,78-0,98

Mortalidad: RR 0,89 IC 0,83-0,96 ACV/ embolismo: RR 0,77 IC 0,70-0,86 HIC: RR 0,46 IC 0,39-0,56

HGI: OR 1,16 IC 1,00-1,34

Mortalidad: RR 0,91 IC 0,85-0,96 ACV/

Embolismo: RR 0,82 IC 0,69-0,98

ACV/embolismo: HR 0,92; 95\% IC 0,84-1,01; $\mathrm{P}=0,066$

HIC: HR 0,44; 0,34-0,59; P<0,001 HGI: HR 1,23; $1,01-1,50 ; \mathrm{P}=0,041$

Mortalidad: RR 0,85 IC 0,76-0,93* ACV/ embolismo: RR 0,93 IC 0,83-1,04*0,79 IC 0,66-

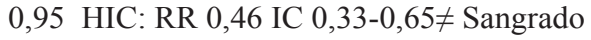
mayor: RR 0,75 IC 0,58-0,96* Sangrado mayor: RR 0,86 IC 0,75-0,99ł

HGI: (todos) RR 1,13 IC 1,02-1,25 p=0,0017

Sangrado mayor: ( $\mathrm{CrCl} 50-80)$ RR 0,84 IC $95 \%$ $0,78-0,91$

Mortalidad: (todos) RR 0,78-0.82 HIC:

(rivaroxabán) RR 0,73 otros NOAC RR 0,39-0,46 HGI: (dabigatrán) RR 1,51

Mortalidad: RR 0,90 IC 0,85-0,95 ACV/ embolismo: RR 0,86 IC 0,75-0,99 HIC: RR 0,48 IC 0,41-0,56 HGI: RR 1,24 IC 1,10-1,39 Sangrado mayor: RR 0,78 IC 0,64-0,94

$x \quad$ HIC: RR 0,40 IC 0,27-0,59 HGI: RR 1,51 IC $1,23-1,84$ 


\begin{tabular}{|c|c|c|c|c|c|c|c|c|}
\hline Estudio & 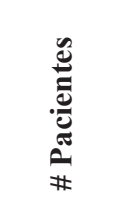 & $\frac{\sqrt[\pi]{\pi}}{\frac{\pi}{\pi}}$ & 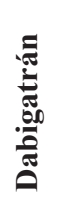 & 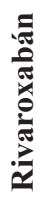 & 焉 & 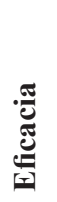 & 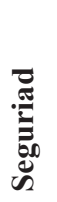 & $\begin{array}{l}\text { Mortalidad /ACV/embolismo /HIC/HGI/ } \\
\text { Sangrado mayor }\end{array}$ \\
\hline & & & & & & & & Sangrado mayor: RR 0,92 IC 0,81-1,05 \\
\hline Sardar P y col (49) & & $\mathrm{x}$ & $\mathrm{x}$ & $\mathrm{x}$ & & $\mathrm{x}$ & $\mathrm{x}$ & HIC: OR 0,42 IC 0,25-0,70 \\
\hline Chatterjee y col (50) & 57491 & $\mathrm{x}$ & $\mathrm{x}$ & $\mathrm{x}$ & & & $\mathrm{x}$ & HIC: OR 0,49 IC 0,36-0,65 \\
\hline Savarese y col (15) & 55011 & $\mathrm{x}$ & $\mathrm{x}$ & $\mathrm{x}$ & $\mathrm{x}$ & $\mathrm{x}$ & $\mathrm{x}$ & $\begin{array}{l}\text { Mortalidad: RR 1,70 IC 1,31-2,19 HIC: RR 0,74 } \\
\text { IC 0,63-0,88 }\end{array}$ \\
\hline Caldeira y col (51) & 100324 & $\mathrm{x}$ & $\mathrm{x}$ & $\mathrm{x}$ & $\mathrm{x}$ & & $\mathrm{x}$ & Sangrado mayor: OR 0,53 IC 0,42-0,68 \\
\hline Capodanno y col (52) & 50578 & $\mathrm{x}$ & $\mathrm{x}$ & $\mathrm{x}$ & & $\mathrm{x}$ & $\mathrm{x}$ & $\begin{array}{l}\text { Mortalidad: OR } 0,88 \text { IC } 0,82-0,95 \text { ACV/ } \\
\text { embolismo: OR 0,82 IC 0,74-0,91 HIC: OR 0,46 } \\
\text { IC 0,38-0,55 HGI: OR 1,7 IC 1,47-1,96 }\end{array}$ \\
\hline Tawfik \& col (53) & 96826 & $\mathrm{x}$ & $\mathrm{x}$ & $\mathrm{x}$ & $\mathrm{x}$ & $\mathrm{x}$ & $\mathrm{x}$ & $\begin{array}{l}\text { ACV/embolismo: RR 0,65 IC 0,52-0,82¥ ACV/ } \\
\text { embolismo: RR 0,82 IC 0,69-0,97× Sangrado } \\
\text { mayor: RR 0,69 IC 0,6-0,8 } \mathrm{f}\end{array}$ \\
\hline Giugliano \& col (54) & & $\mathrm{x}$ & $\mathrm{x}$ & $\mathrm{x}$ & $\mathrm{x}$ & $\mathrm{x}$ & $\mathrm{x}$ & $\begin{array}{l}\text { ACV/embolismo: ACV similar altas dosis } \\
\text { edoxabán }\end{array}$ \\
\hline
\end{tabular}

ACV: Accidente cerebro vascular. OR: Odds Ratio, IC: Intervalo de confianza, RR: Riesgo relativo, HIC: Hemorragia intra-craneal, HIG: Hemorragia gastrointestinal.

con dabigatrán $150 \mathrm{mg}$ en pacientes diabéticos comparado con no diabéticos HR: 0,61 (IC $95 \%$ 0,41-0,91); Hart y $\mathrm{Col}$ (20) reportaron disminución del riesgo de HIC con las dos dosis de dabigatrán D150 RR: 0,40 (IC95 \% 0,27-0,59 $\mathrm{P}<0,001)$ y D110 RR: 0,30 (IC $95 \%$ 0,19-0,45) P<0,001; Eikelboom y Col (21) además mostró reducción del riesgo de HIC RR: 0,30 (IC95 \% 0,19-0,45 P<0,001), RR: 0,42 (IC $95 \%$ 0,29-0,62 P<0,001) y aumento de riesgo de hemorragia gastrointestinal (HGI) con dabigatrán 150 RR: 1,49 (IC 95 \% 1,21-1,84 P<0,001); Bytzer y Col (22) mostraron aumento de riesgo de eventos adversos gastrointestinales con dabigatrán 150 (enfermedad por reflujo gastroesofágico, dispepsia y síntomas GI) RR: 5,06 (IC 95 \% 2,80-9-15 P<0,001), RR: 3,2 (IC95 \% 2,08-5,00 P<0,001), RR: 2,34 (IC $95 \%$ 1,90-2,88 P<0,001) respectivamente.

De los subanálisis incluidos para edoxabán solo 3 mostraron resultados estadísticamente significativos. El estudio de Ruff y Col (23), reportó disminución del riesgo de hemorragia intracraneal (HIC) HR: 0,11 (IC 95 \% 0,04$0,28 \mathrm{p}=0,011$ ), sangrado mayor (altas dosis y bajas dosis) HR: 0,63 (IC $95 \%$ 0,50-0,81 p=0,023), HR: 0,31 (IC 95\% 0,23-0,42 $\mathrm{p}=0,002)$; Steffel y Col (24); reportaron disminución del riesgo de ACV en pacientes en tratamiento con amiodarona a bajas dosis de edoxabán frente aquellos que no HR: 0,60 (IC 95 \% 0,36-0,99 p<0,01); O’Donoghue y Col (25), reportaron disminución del riesgo de ACV en pacientes sin tratamiento previo asignados a altas dosis de edoxabán; pero, aumento en el riesgo de ACV de aquellos pacientes usuarios previos de warfarina con edoxabán a bajas dosis sin tratamiento previo HR: 0,71 (IC 95\% 0,56-0,90 p=0,028), HR: 1,31 (IC $95 \%$ 1,08-1,6 p=0,019) respectivamente.

Para rivaroxabán 5 artículos mostraron resultados estadísticamente significativos: Jones y Col (26), reportaron aumento del riesgo de sangrado mayor y menor en pacientes con enfermedad arterial periférica tratados con rivaroxabán en comparación con warfarina HR: 1,40 (IC 95 \% 1,06-1,86 $\mathrm{P}=0,037)$; Mahaffey y $\mathrm{Col}$ (27), reportaron aumento del riesgo de $\mathrm{ACV} /$ embolismo sistémico en aquellos pacientes asignados a rivaroxabán que al final del estudio pasaron a tratamiento con warfarina HR: 3,72 (IC 95 \% 1,51-9,16 $\mathrm{P}=0,004)$, Sherwood y Col (28); reportaron aumento del sangrado GI con rivaroxabán HR: 1,66 (IC 95 \% 1,34-2,05 $\mathrm{P}<0,0001)$ y además reportaron predictores de sangrado GI que son: anemia al inicio del estudio HR: 1,70 (IC 95\% 1,41-2,04 P<0,0001), sangrado GI previo HR: 2,11 (IC 95 $\%$ 1,62-2.76) y edad mayor (por cada 5 años mayor) HR: 1,11 (IC 95 \% 1,06-1,17 P<0,0001); Hankey y Col (29) 
Tabla 2. Subanálisis incluidos

\begin{tabular}{|c|c|c|c|c|}
\hline Estudio-Autor & Resumen & 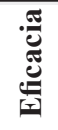 & 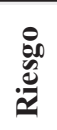 & Resultados \\
\hline \multicolumn{5}{|l|}{ Apixabán } \\
\hline Vinereanu D y col (16) & Según sexo y FA & $\mathrm{X}$ & $\mathrm{X}$ & $\begin{array}{l}\text { Mujeres vs. hombres: muerte de cualquier causa: HR } 0,63 \\
(0,55-0,73 \text {; IC } 95 \%) \mathrm{P}<0,0001 \text { Muerte cardiovascular: HR } \\
0,62(0,51-0,75 ; \text { IC } 95 \%) \mathrm{P}<0,0001\end{array}$ \\
\hline \multirow[t]{3}{*}{ Lopes y Col (17) } & \multirow[t]{3}{*}{ Según riesgo de $\mathrm{ACV}$} & \multirow[t]{3}{*}{$\mathrm{X}$} & \multirow[t]{3}{*}{$\mathrm{X}$} & Apixabán vs. warfarina por CHADS2 y CHA2DS2VASc: \\
\hline & & & & $\begin{array}{l}\text { Sangrado intracraneal: HR 0,42 }(0,30-0,58 \text {; IC } 95 \%) \\
\text { P<0,0001 Apixabán vs. warfarina por CHADS2 y } \\
\text { CHA2DS2VASc: Sangrado mayor: HR 0,69 (0,60-0; IC } \\
\text { 95\%) P<0,0001 apixabán vs. warfarina por CHADS2 y } \\
\text { CHA2DS2VASc: }\end{array}$ \\
\hline & & & & Cualquier sangrado: HR 0,71 (0,68-0,75; IC $95 \%) \mathrm{P}<0,0001$ \\
\hline Hylek EM y col (18) & Sangrado mayor & & $\mathrm{X}$ & $\begin{array}{l}\text { Sangrado mayor seguido de muerte a los } 30 \text { días: HR } 0,50 \\
(0,33-0,74 \text {; IC } 95 \%) \mathrm{P}<0,001 \text { Sangrado intracraneal: } 0,42 \\
(0,30-0,58 \text {; IC } 95 \%) \mathrm{P}<0,001\end{array}$ \\
\hline Hohnloser y col (55) & Según función renal & $\mathrm{X}$ & $\mathrm{X}$ & $\begin{array}{l}\text { Sangrado mayor: menor en todos los intervalos de función } \\
\text { renal, mayor con: eGFR }<50 \mathrm{ml} / \mathrm{min} \text { HR } 0,50 \text { IC } 0,38-0,66 \\
\mathrm{p}=0,005 \text { CKD-EPI HR } 0,48 \text { IC } 0,37-0,64 \mathrm{p}=0,003\end{array}$ \\
\hline \multicolumn{5}{|l|}{ Dabigatrán } \\
\hline \multirow[t]{2}{*}{ Brambatti M y col (19) } & Diabetes mellitus y FA & $\mathrm{X}$ & $\mathrm{X}$ & $\begin{array}{l}\text { Pacientes con DM y sin DM D150 HR 0,61 IC 0,41-0,91 } \\
\text { Hart RG y col (20) }\end{array}$ \\
\hline & Hemorragia intracraneal & & $\mathrm{X}$ & $\begin{array}{l}\text { HIC: dabigatrán } 150 \text { vs. warfarina: RR } 0,40(0,27-0,59 \\
\text { IC95 \%) P<0,001 dabigatrán } 110 \text { vs. warfarina: RR 0,30 } \\
(0,19-0,45 \text {; IC } 95 \%) \mathrm{P}<0,001\end{array}$ \\
\hline \multirow[t]{2}{*}{ Eikelboom JW y col (21) } & \multirow[t]{2}{*}{$\begin{array}{l}\text { Sangrado con } 2 \text { dosis en } \\
\text { menores y mayores de } 75 \text { años }\end{array}$} & \multirow[t]{2}{*}{$\mathrm{X}$} & & $\begin{array}{l}\text { HIC: dabigatrán } 110 \text { vs. warfarina: RR } 0,30(0,19-0,45 \\
\text { IC } 95 \%) \text { P }<0,001 \text { dabigatrán } 150 \text { vs. warfarina: RR } 0,42 \\
(0,29-0,62 \text {; IC } 95 \%) \mathrm{P}<0,001\end{array}$ \\
\hline & & & & $\begin{array}{l}\text { HGI: dabigatrán } 150 \text { vs. warfarina: RR 1,49 (1,21-1,84; IC } \\
95 \%) \mathrm{P}<0,001\end{array}$ \\
\hline Bytzer P y col (22) & Eventos adversos GI & & $\mathrm{X}$ & $\begin{array}{l}\text { ERGE: RR 5,06 (2,80-9-15; IC } 95 \%) \mathrm{P}<0,001 \text { Dispepsia: } \\
\text { RR 3,2 (2,08-5,00; IC95 \%) P<0,001 Síntomas GI: RR 2,34 } \\
(1,90-2,88 ; \text { IC } 95 \%) \mathrm{P}<0,001\end{array}$ \\
\hline Wallentin y col (56) & Relación con INR & $\mathrm{X}$ & $\mathrm{X}$ & $\begin{array}{l}\text { Sangrado mayor: TTRc }<57,1 \text { \% D150 HR 0,71 IC 0,52- } \\
0,96 \mathrm{p}=0,03\end{array}$ \\
\hline \multicolumn{5}{|l|}{ Edoxabán } \\
\hline Ruff y col (23) & $\begin{array}{l}\text { Dosis, concentración, } \\
\text { actividad del factor Xa y }\end{array}$ & $\mathrm{X}$ & $\mathrm{X}$ & $\begin{array}{l}\text { HIC: HR } 0,11 \text { IC } 0,04-0,28 p=0.011 \text { Sangrado mayor: Alta } \\
\text { resultados dosis: sangrado mayor HR } 0,63 \text { IC } 0,50-0,81 \\
p=0,023 \text { baja dosis: HR } 0,31 \text { IC } 0,23-0,42 p=0,002\end{array}$ \\
\hline Steffel y col (24) & FA con amiodarona & $\mathrm{X}$ & $\mathrm{X}$ & $\begin{array}{l}\text { ACV: con amiodarona baja dosis vs. warfarina ACV: HR } \\
0,60 \text { IC } 0,36-0,99 p<0,01\end{array}$ \\
\hline O’Donoghue y col (25) & Uso previo de warfarina & $\mathrm{X}$ & $\mathrm{X}$ & $\begin{array}{l}\text { ACV: alta dosis naive HR } 0,71 \text { IC } 0,56-0,90 p=0,028 \text { uso } \\
\text { previo bajas dosis HR } 1,31 \text { IC } 1,08-1,6 p=0,019\end{array}$ \\
\hline \multicolumn{5}{|l|}{ Rivaroxabán } \\
\hline Jones WS y col (26) & $\begin{array}{l}\text { Sangrado según enfermedad } \\
\text { arterial periférica y FA }\end{array}$ & $\mathrm{X}$ & $\mathrm{X}$ & $\begin{array}{l}\text { Sangrado mayor o menor: HR 1,40 (1,06-1,86; IC } 95 \%) \\
\mathrm{P}=0,037\end{array}$ \\
\hline Mahaffey y col (27) & $\begin{array}{l}\text { Uso de warfarina al final del } \\
\text { estudio }\end{array}$ & & $\mathrm{X}$ & ACV: HR 3,72 (1,51-9,16; IC $95 \%) \mathrm{P}=0,004$ \\
\hline Sherwood MW y col (28) & Sangrado GI en FA & & $\mathrm{X}$ & $\begin{array}{l}\text { HGI: HR 1,66 (1,34-2,05; IC } 95 \%) \mathrm{P}<0,0001 \text { Predictores de } \\
\text { sangrado GI: anemia al inicio: HR } 1,70(1,41-2,04) \\
\mathrm{P}<0,0001 \text { Sangrado GI previo: HR 2,11 }(1,62-2,76) \\
\mathrm{P}<0,0001 \text { Edad (por cada } 5 \text { años mayor): HR } 1,11(1,06- \\
1,17) \mathrm{P}<0,0001\end{array}$ \\
\hline
\end{tabular}


Tabla 2. Subanálisis incluidos

\begin{tabular}{|c|c|c|c|c|}
\hline Estudio-Autor & Resumen & 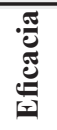 & 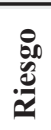 & Resultados \\
\hline Hankey GJ y col (29) & Sangrado intracraneal & & $\mathrm{X}$ & HIC: HR 0,60 (0,44-0,82; IC 95\%) P0,001 \\
\hline \multirow[t]{2}{*}{ Goodman SG y col (30) } & $\begin{array}{l}\text { Factores asociados a sangrado } \\
\text { mayor }\end{array}$ & & $\mathrm{X}$ & $\begin{array}{l}\text { Sangrado mayor: edad: HR 1,17 (1,12-1,23; IC } 95 \%) \\
\mathrm{P}<0,0001\end{array}$ \\
\hline & & & & $\begin{array}{l}\text { PAD }<90 \text { mmHg: } 1,28(1,11-1,47 \text {; IC } 95 \%) \mathrm{P}<0,0001 \\
\text { Historia de sangrado GI: HR 1,88 (1,44-2,45; IC } 95 \%) \\
\mathrm{P}<0,0001 \text { Uso de aspirina: } 1,42(1,23-1,64 ; \text { IC } 95 \%) \\
\mathrm{P}<\text {, } 0001 \text { anemia: } 1,88(1,59-2,22 ; \text { IC } 95 \%) \mathrm{P}<0,0001\end{array}$ \\
\hline
\end{tabular}

FA: Fibrilación auricular, HR: Hazard Ratio, RR: Riesgo relativo, ACV: Accidente cerebro vascular, DM: diabetes mellitus, HIC: hemorragia intra-craneal, HGI: hemorragia gastrointestinal, GI: gastrointestinal(es), TTRc: Tiempo en rango terapéutico.

reportaron disminución del riesgo de $\mathrm{HIC}$ con rivaroxabán HR: 0,60 (IC 95 \% 0,44-0,82 P=0,001); Goodman y Col (30) reportaron aumento de sangrado mayor HR: 1,17 (IC $95 \%$ 1,12-1,23 $\mathrm{P}<0,0001)$, predictores de sangrado mayor los cuales fueron: historia de sangrado GI HR: 1,88 (IC 95 $\%$ 1,44-2,45 P<0,.0001), uso de aspirina 1,42 (IC $95 \% 1,23$ $1,64 \mathrm{P}<0,0001)$ y anemia 1,88 (IC $95 \% 1,59-2,22 \mathrm{P}<0,0001)$.

\section{DISCUSIÓN}

Los nuevos anticoagulantes orales en general reducen el riesgo de ACV/embolismo sistémico, riesgo de muerte, hemorragia intracraneal y sangrado mayor, pero aumentan el riesgo de hemorragia gastrointestinal; estos resultados de los meta-análisis basados en los ensayos clínicos pivótales y cuando se evaluaban en conjunto. Existen particularidades para cada uno de los medicamentos incluidos como: disminución del riesgo de muerte $\mathrm{ACV} /$ embolismo sistémico, HIC y sangrado mayor con apixabán, dabigatrán y rivaroxabán cuando el tiempo en rango terapéutico de warfarina era menor del $65 \%$ del tiempo, así mismo reducción del riesgo de sangrado mayor en pacientes con función renal moderada, aumento del riesgo de muerte y disminución de HIC en pacientes con falla renal.

Los subanálisis reportaron disminución en ciertas variables para medicamentos en particular como: para apixabán disminución del riesgo de sangrado intracraneal y sangrado mayor en relación con el riesgo de $\mathrm{ACV}$, disminución del riesgo de muerte a los 30 días y sangrado intracraneal; para dabigatrán disminución del riesgo de ACV/embolismo sistémico en pacientes diabéticos, disminución del riesgo de HIC con las dos dosis de dabigatrán, pero aumento de riesgo de HGI y síntomas gastrointestinales. Para edoxabán hubo disminución de riesgo de sangrado mayor, ACV/ embolismo sistémico en pacientes en tratamiento con amiodarona y aquellos nuevos usuarios de anticoagulación oral con edoxabán a altas dosis, pero hubo aumento del riesgo de $\mathrm{ACV} /$ embolismo sistémico en pacientes con uso previo de warfarina y asignados a edoxabán a bajas dosis. Para rivaroxabán hubo aumento del riesgo de sangrado mayor y menor en pacientes con enfermedad arterial periférica, aumento de riesgo de $\mathrm{ACV} /$ embolismo sistémico en pacientes quienes durante el estudio fueron asignados a rivaroxabán y que al final pasaron a warfarina, mostró aumento del riesgo de sangrado gastrointestinal y sangrado mayor con rivaroxabán.

Es importante tener en cuenta que todos los meta-análisis y subanálisis están basados en los ensayos clínicos pivótales, estos estudios fueron ensayos clínicos aleatorizados de no inferioridad, en los cuales se expone como hipótesis que los nuevos anticoagulantes orales son al menos tan eficaces como el tratamiento estándar con warfarina, esta aseveración a priori es importante para la fijación del delta que en ningún caso tuvo como objetivo encontrar diferencias clínicamente significativas frente al status quo. A pesar de estas consideraciones los nuevos anticoagulantes orales son reconocidos en las diferentes guías de manejo de FA, con algunas consideraciones sobre el sangrado gastrointestinal.

Existe tendencia al aumento del sangrado gastrointestinal en los pacientes incluidos en los ensayos clínicos para dabigatrán y rivaroxabán en fibrilación auricular, tendencia que no se evidencia en los ensayos clínicos para estos dos medicamentos en la prevención de trombosis venosa profunda, la posible explicación a esta diferencia es debida a la edad de los pacientes incluidos en RE-LY y ROCKET-AF 71 y 73 años respectivamente, frente a la edad promedio de los pacientes en los ensayos clínicos para embolismo venoso y pulmonar que fue de 60 años; al parecer por disminución del aclaramiento renal debido a la edad que afecta más a dabigatrán y rivaroxabán que a warfarina (31). Dos subanálisis analizaron los factores de riesgo asociados al sangrado mayor en pacientes en tratamiento con rivaroxabán (Sherwood MW y col (28), 
Goodman SG y col (30)) los autores reportaron: anemia al inicio del estudio, sangrado gastrointestinal previo, la edad y el uso de aspirina; como factores predictores que hay que considerarlos al momento de iniciar tratamiento con cualquier anticoagulante oral.

Consideraciones como la anterior, el amplio conocimiento del farmacocinética y farmacodinamia de warfarina, así como el conocido y fácil uso de antídoto en caso de sangrado por sobre-anticoagulación y consideraciones económicas que van desde análisis de impacto presupuestario hasta estudios costo-efectividad de los nuevos anticoagulantes orales comparados con warfarina, hacen que aun siga siendo considerada de primera línea en la mayoría de las guías de manejo de FA. $(32,33)$. Existe necesidad de ensayos clínicos pragmáticos y grandes estudios retrospectivos usando información de grandes bases de datos (Big-Data) que soporten aún más el uso de los nuevos anticoagulantes orales en condiciones de práctica diaria como poblaciones en edades extremas, uso fuera de indicación aprobada y posiblemente nuevos hallazgos relacionados con la seguridad que solo son descritos en condiciones de uso fuera de los ensayos clínicos.

La valoración riesgo-beneficio y la decisión en anticoagulación debe ser individualizada y compartida entre el médico y el paciente, teniendo en cuenta los diferentes factores de riesgo, antecedentes patológicos, la tolerabilidad, tiempo de rango terapéutico del INR, potenciales interacciones farmacológicas y antecedentes de toma de anticoagulantes orales. En los pacientes que tienen trata- miento con warfarina, y que están estables, su condición se controla fácilmente y están satisfechos con este manejo, no es necesario cambiar a un nuevo anticoagulante oral, sin embargo, es importante tener informado al paciente de las diferentes opciones con las que cuenta. Recalcamos la importancia en la práctica clínica diaria de la realización de un simple balance entre los riesgos y beneficios de cualquier intervención para elegir el tratamiento que mejor se adecua a cada paciente en particular.

\section{CONCLUSIÓN}

Los nuevos anticoagulantes orales son más efectivos y con mejor perfil de seguridad comparado con warfarina, con particularidades para cada uno en los subanálisis, que en su mayoría no alcanzaron significancia estadística en los subgrupos de pacientes estudiados. Comparaciones directas, en las condiciones de prescripción en práctica clínica son necesarias para decidir cuál de los nuevos anticoagulantes muestra un mejor perfil riesgo-beneficio.

\section{Agradecimientos}

Red Cardiecol, Colciencias código: 5020-53-731809

El estudio no contó con financiación alguna

\section{Conflicto de intereses}

Los autores manifiestan no tener conflictos de intereses en este estudio.

\section{REFERENCIAS}

1. Ahmad Y, Lip G Y, Lane DA. Recent developments in understanding epidemiology and risk determinants of atrial fibrillation as a cause of stroke. Can J Cardiol. 2013;29(7):S4-S13. http://dx.doi.org/10.1016/j. cjca.2013.03.009

2. Andersen LV, Vestergaard P, Deichgraeber P, Lindholt JS, Mortensen LS, Frost L. Warfarin for the prevention of systemic embolism in patients with non-valvular atrial fibrillation: a meta-analysis. Heart. 2008;94(12):1607-13. http://dx.doi.org/10.1136/hrt.2007.135657

3. DeWilde S, Carey IM, Emmas C, Richards N, Cook DG. Trends in the prevalence of diagnosed atrial fibrillation, its treatment with anticoagulation and predictors of such treatment in UK primary care. Heart. 2006;92(8):106470. http://dx.doi.org/10.1136/hrt.2005.069492

4. Gallego P, Roldan V, Marin F, Romera M, Valdes M, Vicente $\mathrm{V}$, et al. Cessation of oral anticoagulation in relation to mortality and the risk of thrombotic events in patients with atrial fibrillation. Thromb Haemost.
2013;110(6):1189-98. http:/ /dx.doi.org/10.1160/TH1307-0556

5. Friberg L, Benson L, Rosenqvist M, Lip GY. Assessment of female sex as a risk factor in atrial fibrillation in Sweden: nationwide retrospective cohort study. BMJ. 2012;344:e3522. https://doi.org/10.1136/bmj.e3522

6. Hsu JC, Hsieh C, Yang YK, Lu CY. Net clinical benefit of oral anticoagulants: a multiple criteria decision analysis. PloS one. 2015;10(4):e0124806. https://doi. org/10.1371/journal.pone.0124806

7. January CT, Wann LS, Alpert JS, Calkins H, Cigarroa JE, Conti JB, et al. 2014 AHA/ACC/HRS guideline for the management of patients with atrial fibrillation: a report of the American College of Cardiology/ American Heart Association Task Force on Practice Guidelines and the Heart Rhythm Society. J Am Coll Cardiol. 2014;64(21):e1-e76. https://doi.org/10.1016/j. jacc.2014.03.022 
8. Kirchhof P, Benussi S, Kotecha D, Ahlsson A, Atar D, Casadei B, et al. 2016 ESC Guidelines for the management of atrial fibrillation developed in collaboration with EACTS The Task Force for the management of atrial fibrillation of the European Society of Cardiology (ESC) Developed with the special contribution of the European Heart Rhythm Association (EHRA) of the ESC Endorsed by the European Stroke Organisation (ESO). Eur J Cardiothorac Surg. 2016;50(5):e1-e88. https://doi.org/10.1093/ejcts/ezw313

9. Baker WL, Phung OJ. Systematic review and adjusted indirect comparison meta-analysis of oral anticoagulants in atrial fibrillation. Circ Cardiovasc Qual Outcomes. 2012;5(5):711-19. https://doi.org/10.1161/CIRCOUTCOMES.112.966572

10. Romanelli RJ, Nolting L, Dolginsky M, Kym E, Orrico KB Dabigatran Versus Warfarin for Atrial Fibrillation in Real-World Clinical Practice A Systematic Review and Meta-Analysis. Circ Cardiovasc Qual Outcomes. 2016;9(2): 126-134. https://doi.org/10.1161/CIRCOUTCOMES.115.002369

11. Bloom BJ, Filion KB, Atallah R, Eisenberg MJ. Meta-analysis of randomized controlled trials on the risk of bleeding with dabigatran. Am J Cardiol. 2014;113(6):1066-74. https://doi.org/10.1016/j.amjcard.2013.11.049

12. Wasserlauf G, Grandi SM, Filion KB, Eisenberg MJ. Meta-analysis of rivaroxaban and bleeding risk. Am J Cardiol. 2013;112(3):454-60. https://doi.org/10.1016/j. amjcard.2013.03.054

13. Gómez-Outes A, Terleira-Fernández AI, Calvo-Rojas G, Suárez-Gea ML, Vargas-Castrillón E. Dabigatran, rivaroxaban, or apixaban versus warfarin in patients with nonvalvular atrial fibrillation: a systematic review and metaanalysis of subgroups. Thrombosis. 2013;2013:640-723. https://doi.org/10.1155/2013/640723

14. Raccah BH, Perlman A, Danenberg HD, Pollak A, Muszkat M, Matok I. Major Bleeding and Hemorrhagic Stroke with Direct Oral Anticoagulants in Patients with Renal Failure: Systematic Review and Meta-Analysis of Randomized Trials. CHEST. 2016;149(6):1516-24. https://doi.org/10.1016/j.chest.2015.12.029

15. Savarese G, Giugliano RP, Rosano GM, McMurray J, Magnani G, Filippatos $G$, et al. Efficacy and safety of novel oral anticoagulants in patients with atrial fibrillation and heart failure: a meta-analysis. JACC Heart Fail. 2016;4(11):870-80. . https://doi.org/10.1016/j. jchf.2016.07.012

16. Vinereanu D, Stevens SR, Alexander JH, Al-Khatib SM, Avezum A, Bahit MC, et al. Clinical outcomes in patients with atrial fibrillation according to sex during anticoagulation with apixaban or warfarin: a secondary analysis of a randomized controlled trial. Eur Heart J. 2015;36(46):3268-75. https://doi.org/10.1093/eurheartj/ehv447

17. Lopes RD, Al-Khatib SM, Wallentin L, Yang H, Ansell J, Bahit MC, et al. Efficacy and safety of apixaban compared with warfarin according to patient risk of stroke and of bleeding in atrial fibrillation: a secondary analysis of a randomised controlled trial. Lancet. 2012;380(9855):1749-58. https://doi.org/10.1016/
S0140-6736(12)60986-6

18. Hylek EM, Held C, Alexander JH, Lopes RD, De Caterina R, Wojdyla DM, et al. Major bleeding in patients with atrial fibrillation receiving apixaban or warfarin: The ARISTOTLE Trial (Apixaban for Reduction in Stroke and Other Thromboembolic Events in Atrial Fibrillation): Predictors, Characteristics, and Clinical Outcomes. J Am Coll Cardiol. 2014;63(20):2141-47. https://doi. org/10.1016/j.jacc.2014.02.549

19. Brambatti M, Darius H, Oldgren J, Clemens A, Noack $\mathrm{HH}$, Brueckmann $\mathrm{M}$, et al. Comparison of dabigatran versus warfarin in diabetic patients with atrial fibrillation: Results from the RE-LY trial. Int J Cardiol. 2015;196:12731. https://doi.org/10.1016/j.ijcard.2015.05.141

20. Hart RG, Diener HC, Yang S, Connolly SJ, Wallentin L, Reilly PA, et al. Intracranial hemorrhage in atrial fibrillation patients during anticoagulation with warfarin or dabigatran the RE-LY trial. Stroke. 2012;43(6):1511-17. https://doi.org/10.1161/STROKEAHA.112.650614

21. Eikelboom JW, Wallentin L, Connolly SJ, Ezekowitz M, Healey JS, Oldgren J, et al. Risk of bleeding with 2 doses of dabigatran compared with warfarin in older and younger patients with atrial fibrillation an analysis of the randomized evaluation of long-term anticoagulant therapy (RE-LY) trial. Circulation. 2011;123(21):2363-72. https://doi.org/10.1161/CIRCULATIONAHA.110.00474

22. Bytzer P, Connolly SJ, Yang S, Ezekowitz M, Formella S, Reilly PA, et al. Analysis of upper gastrointestinal adverse events among patients given dabigatran in the RE-LY trial. Clin Gastroenterol Hepatol. 2013;11(3):246-52. https://doi.org/10.1016/j.cgh.2012.10.021

23. Ruff CT, Giugliano RP, Braunwald E, Morrow DA, Murphy SA, Kuder JF, et al. Association between edoxaban dose, concentration, anti-Factor Xa activity, and outcomes: an analysis of data from the randomised, double-blind ENGAGE AF-TIMI 48 trial. Lancet. 2015;385(9984):2288-95. https://doi.org/10.1016/ S0140-6736(14)61943-7

24. Steffel J, Giugliano RP, Braunwald E, Murphy SA, Atar $\mathrm{D}$, Heidbuchel $\mathrm{H}$, et al. Edoxaban vs. warfarin in patients with atrial fibrillation on amiodarone: a subgroup analysis of the ENGAGE AF-TIMI 48 trial. Eur Heart J. 2015;36(33):2239-2245. https://doi.org/10.1093/ eurheartj/ehv201

25. O’Donoghue ML, Ruff CT, Giugliano RP, Murphy SA, Grip LT, Mercuri MF, et al. Edoxaban vs. warfarin in vitamin $\mathrm{K}$ antagonist experienced and naive patients with atrial fibrillation. Eur Heart J. 2015;36(23):1470-1477. https://doi.org/10.1093/eurheartj/ehv014

26. Jones WS, Hellkamp AS, Halperin J, Piccini JP, Breithardt $G$, Singer DE, et al. Efficacy and safety of rivaroxaban compared with warfarin in patients with peripheral artery disease and non-valvular atrial fibrillation: insights from ROCKET AF. Eur Heart J. 2013;35(4):242-249. https:/ / doi.org/10.1093/eurheartj/eht492

27. Mahaffey KW, Wojdyla D, Hankey GJ, White HD, Nessel CC, Piccini JP, et al. Clinical outcomes with rivaroxaban in patients transitioned from vitamin $K$ antagonist therapy: a subgroup analysis of a randomized trial. 
Ann Intern Med. 2013;158(12):861-868. https://doi. org/10.7326/0003-4819-158-12-201306180-00003.

28. Sherwood MW, Nessel CC, Hellkamp AS, Mahaffey KW, Piccini JP, Suh EY, et al. Gastrointestinal bleeding in patients with atrial fibrillation treated with rivaroxaban or warfarin: ROCKET AF trial. J Am Coll Cardiol. 2015;66(21):2271-2281. . https://doi.org/10.1016/j. jacc.2015.09.024.

29. Hankey GJ, Patel MR, Stevens SR, Becker RC, Breithardt G, Carolei A, et al. Rivaroxaban compared with warfarin in patients with atrial fibrillation and previous stroke or transient ischaemic attack: a subgroup analysis of ROCKET AF. Lancet Neurol. 2012;11(4):315-22. https://doi.org/10.1016/S1474-4422(12)70042-X

30. Goodman SG, Wojdyla DM, Piccini JP, White HD, Paolini JF, Nessel CC, et al. Factors associated with major bleeding events: insights from the ROCKET AF trial (rivaroxaban once-daily oral direct factor Xa inhibition compared with vitamin $\mathrm{K}$ antagonism for prevention of stroke and embolism trial in atrial fibrillation). J Am Coll Cardiol. 2014;63(9):891-900. https://doi.org/10.1016/j. jacc.2013.11.013

31. Sarrazin MV, Rose A. Safety of new oral anticoagulants. BMJ. 2015;350:h1679. https://doi.org/10.1136/bmj. h1679

32. Amin A, Deitelzweig S, Jing Y, Makenbaeva D, Wiederkehr D, Lin J, et al. Estimation of the impact of warfarin's time-in-therapeutic range on stroke and major bleeding rates and its influence on the medical cost avoidance associated with novel oral anticoagulant use-learnings from ARISTOTLE, ROCKET-AF, and RE-LY trials. J Thromb Thrombolysis. 2015;38(2):150159. https://doi.org/10.1007/s11239-013-1048-z

33. Connock M, Stevens C, Fry-Smith A, Jowett S, Fitzmaurice $\mathrm{D}$, Moore $\mathrm{D}$, et al. Clinical effectiveness and costeffectiveness of different models of managing long-term oral anticoagulation therapy: a systematic review and economic modelling. Health Technol Assess. 2007;1138:66.

34. Bruins Slot KM, Berge E. Factor Xa inhibitors versus vitamin $K$ antagonists for preventing cerebral or systemic embolism in patients with atrial fibrillation. Stroke. 2013;44(12):e165-e167. https://doi. org/10.1002/14651858.CD008980

35. Assiri A, Al-Majzoub O, Kanaan AO, Donovan JL, Silva M. Mixed treatment comparison meta-analysis of aspirin, warfarin, and new anticoagulants for stroke prevention in patients with nonvalvular atrial fibrillation. Clinic Ther. 2013;35(7):967-984. https://doi.org/10.1016/j. clinthera.2013.05.011

36. Wasserlauf G, Grandi SM, Filion KB, Eisenberg MJ Meta-analysis of rivaroxaban and bleeding risk. Am J Cardiol. 2013;112(3):454-60. . https://doi. org/10.1016/j.amjcard.2013.03.054.

37. Baker WL, Phung OJ. Systematic review and adjusted indirect comparison meta-analysis of oral anticoagulants in atrial fibrillation. Circ Cardiovasc Qual Outcomes. 2012;5(5):711-719. https://doi.org/10.1161/CIRCOUTCOMES.112.966572

38. Testa L, Agnifili M, Latini RA, Mattioli R, Lanotte S, De Marco F, et al. Adjusted indirect comparison of new oral anticoagulants for stroke prevention in atrial fibrillation. QJM. 2012;105(10):949-57. https://doi.org/10.1093/ qjimed/hcs114

39. Miller CS, Grandi SM, Shimony A, Filion KB, Eisenberg MJ. Meta-analysis of efficacy and safety of new oral anticoagulants (dabigatran, rivaroxaban, apixaban) versus warfarin in patients with atrial fibrillation. Am J Cadiol. 2012;110(3):453-60. https://doi.org/10.1016/j. amjcard.2012.03.049

40. Kwong JS, Lam YY, Yan BP, Yu CM. Bleeding of new oral anticoagulants for stroke prevention in atrial fibrillation: a meta-analysis of randomized controlled trials. Cardiovasc Drugs Ther. 2012;27(1):23-35. https://doi. org/10.1007/s10557-012-6426-9

41. Dentali F, Riva N, Crowther M, Turpie AG, Lip GY, Ageno W. Efficacy and safety of the novel oral anticoagulants in atrial fibrillation: a systematic review and meta-analysis of the literature. Circulation. 2012;126(20):2381-91. https://doi.org/10.1161/CIRCULATIONAHA.112.115410

42. Holster IL, Valkhoff VE, Kuipers EJ, Tjwa ET. New oral anticoagulants increase risk for gastrointestinal bleeding: a systematic review and meta-analysis. Gastroenterology. 2013;145(1):105-112. https://doi.org/10.1053/j. gastro.2013.02.041

43. Dogliotti A, Paolasso E, Giugliano RP. Novel oral anticoagulants in atrial fibrillation: a meta $\square$ analysis of large, randomized, controlled trials vs warfarin. Clinic Cardiol. 2013;36(2):61-67. https://doi.org/10.1002/clc.22081

44. Romanelli RJ, Nolting L, Dolginsky M, Kym E, Orrico KB. Dabigatran Versus Warfarin for Atrial Fibrillation in Real-World Clinical Practice A Systematic Review and Meta-Analysis. Circ Cardiovasc Qual Outcomes. 2016;9(2):126-134. https://doi.org/10.1161/CIRCOUTCOMES.115.002369

45. Loffredo L, Perri L, Violi F. Impact of new oral anticoagulants on gastrointestinal bleeding in atrial fibrillation: A meta-analysis of interventional trials. Dig Liver Dis. 2015;47(5):429-431. https://doi.org/10.1016/j. dld.2015.01.159

46. Raccah BH, Perlman A, Danenberg HD, Pollak A, Muszkat M, Matok I. Major Bleeding and Hemorrhagic Stroke with Direct Oral Anticoagulants in Patients with Renal Failure: Systematic Review and Meta-Analysis of Randomized Trials. CHEST. 2016;149(6):1516-24. https://doi.org/10.1016/j.chest.2015.12.029

47. Lin L, Lim WS, Zhou HJ, Khoo AL, Tan KT, Chew AP, et al. Clinical and Safety Outcomes of Oral Antithrombotics for Stroke Prevention in Atrial Fibrillation: A Systematic Review and Network Meta-analysis. J Am Med Dir Assoc. 2015;16(12):1103.e1-1119. https:/ / doi. org/10.1016/j.jamda.2015.09.008

48. Jia B, Lynn HS, Rong F, Zhang W. Meta-analysis of efficacy and safety of the new anticoagulants versus warfarin in patients with atrial fibrillation. J Cardiovasc Pharmacol. 2014;64(4):368-374. https://doi.org/10.1097/ FJC.0000000000000129

49. Sardar P, Chatterjee S, Wu WC, Lichstein E, Ghosh J, Aikat $S$, et al. New oral anticoagulants are not superior to warfarin in secondary prevention of stroke or transient 
ischemic attacks, but lower the risk of intracranial bleeding: insights from a meta-analysis and indirect treatment comparisons. PLoS One. 2013;8(10):e77694. https://doi. org/10.1371/journal.pone.0077694

50. Chatterjee S, Sardar P, Biondi-Zoccai G, Kumbhani DJ. New oral anticoagulants and the risk of intracranial hemorrhage: traditional and Bayesian meta-analysis and mixed treatment comparison of randomized trials of new oral anticoagulants in atrial fibrillation. JAMA Neurol. 2013;70(12):1486-90.

51. Caldeira D, Rodrigues FB, Barra M, Santos AT, de Abreu D, Gonçalves N, et al. Non-vitamin K antagonist oral anticoagulants and major bleeding-related fatality in patients with atrial fibrillation and venous thromboembolism: a systematic review and meta-analysis. Heart. 2015;101(15):1204-1211. https://doi.org/10.1001/ jamaneurol.2013.4021

52. Capodanno D, Capranzano P, Giacchi G, Calvi V, Tamburino C. Novel oral anticoagulants versus warfarin in non-valvular atrial fibrillation: a meta-analysis of 50,578 patients. Int J Cardiol. 2013;167(4):1237-41. https:/ / doi. org/10.1016/j.ijcard.2012.03.148

53. Tawfik A. Systematic review and network meta-analysis of stroke prevention treatments in patients with atrial fibrillation. Comparative and Cost-effectiveness of
Stroke Prevention Treatments in Atrial Fibrillation: A New Generation of Drugs. 2015;21.

54. Giugliano RP, Ruff CT, Braunwald E, Murphy SA, Wiviott SD, Halperin JL, et al. Edoxaban versus warfarin in patients with atrial fibrillation. $\mathrm{N}$ Engl J Med. 2013;369(22):2093-2104. https://doi.org/10.1056/ NEJMoa1310907

55. Hohnloser SH, Hijazi Z, Thomas L, Alexander JH, Amerena J, Hanna M, et al. Efficacy of apixaban when compared with warfarin in relation to renal function in patients with atrial fibrillation: insights from the ARISTOTLE trial. Eur Heart J. 2012;33(22):2821-30. https:/ / doi.org/10.1093/eurheartj/ehs274

56. Wallentin L, Yusuf S, Ezekowitz MD, Alings M, Flather M, Franzosi MG, et al. Efficacy and safety of dabigatran compared with warfarin at different levels of international normalised ratio control for stroke prevention in atrial fibrillation: an analysis of the RE-LY trial. Lancet. 2010;376(9745):975-983. https://doi.org/10.1016/ S0140-6736(10)61194-4

57. van Walraven C, Hart RG, Singer DE, Koudstaal PJ, Connolly S. Oral anticoagulants vs. aspirin for stroke prevention in patients with non-valvular atrial fibrillation: the verdict is in. Card Electrophysiol Rev. 2003;7(4):37478. 